\title{
Acute-on-chronic hepatitis. A case report of autoimmune hepatitis/primary sclerosing cholangitis/ulcerative colitis overlap syndrome in a 15-year-old patient
}

\author{
Aneta Nalepa', Małgorzata Woźniak', Joanna Cielecka-Kuszyk², Marek Stefanowicz³, Irena Jankowska', Maciej Dądalski', \\ Joanna Pawłowska' \\ 'Department of Gastroenterology, Hepatology, Nutrition Disorders and Paediatrics, The Children's Memorial Health Institute, \\ Warsaw, Poland \\ ${ }^{2}$ Department of Pathology, The Children's Memorial Health Institute, Warsaw, Poland \\ ${ }^{3}$ Department of Paediatric Surgery and Organ Transplantation, The Children's Memorial Health Institute, Warsaw, Poland
}

\begin{abstract}
Acute-on-chronic liver failure (ACLF) is a disease in which a rapid deterioration of liver function occurs in patients with chronic liver disease, and is usually associated with a precipitating event. We present the case of a boy with autoimmune hepatitis/primary sclerosing cholangitis/ulcerative colitis (AIH/PSC/UC) overlap syndrome, in whom liver function was stable for 4.5 years of treatment. At 15 years of age the patient was hospitalized due to a deterioration of his general condition, severe abdominal pain, diarrhoea, vomiting and weight loss. There was also a rapid deterioration of liver function and a deterioration of renal function. Despite a wide spectrum of diagnostic examinations, no precipitating agent was found. After two episodes of massive bleeding from the gastrointestinal tract, the patient was transferred to the intensive care unit. The patient underwent a successful liver transplantation. ACLF can cause irreversible liver failure with a high mortality rate, which calls for liver transplantation.
\end{abstract}

Key words: children, liver transplantation, ACLF.

\section{Address for correspondence}

Joanna Pawłowska, Department of Gastroenterology, Hepatology, Nutrition Disorders and Paediatrics, The Children's Memorial Health Institute, 20 Dzieci Polskich Avenue, 04-730 Warsaw, Poland, phone: +48 22815 73 80, e-mail: j.pawlowska@czd.pl

\section{Introduction}

Since 1995 when Ohnishi first used the term "acuteon-chronic liver failure" (ACLF), several definitions have been proposed [1]. Generally, two definitions are currently used. One, proposed by the American Association for the Study of Liver Disease (AASLD) and the European Association for the Study of the Liver (EASL), states that it is an "acute deterioration of pre-existing, chronic liver disease (CLD), usually related to a precipitating event and associated with increased mortality at 3 months due to multisystem organ failure" [2]. The second definition, recommended by the Asian $\mathrm{Pa}$ cific Association for the Study of the Liver (APASL), states that it is an "acute hepatic insult manifesting as jaundice (serum bilirubin $\geq 5 \mathrm{mg} / \mathrm{dl}$ ) and coagulopathy (international normalized ratio [INR] $\geq 1.5$ ), complicated within 4 weeks by ascites and/or encephalopathy in a patient with previously diagnosed or undiagnosed chronic liver disease" [3]. Among "new" insults precipitating liver failure are bacterial or viral infections, drugs or hepatotoxins, severe variceal bleeding and surgery $[2,4]$. Epidemiology of ACLF, both in adult patients and in children, is not well defined. Moreau et al., in a recent study conducted in Europe in 1343 adult patients with acute liver decompensation in the course of underlying liver disease, reported the prevalence of ACLF of $30.9 \%$. Mortality among patients with ACLF is higher (50-90\%) than in patients with CLD awaiting a liver transplantation [5-7]. 
We present the case of a boy with autoimmune hepatitis/primary sclerosing cholangitis (AIH/PSC) overlap syndrome and ulcerative colitis (UC) who developed ACLF.

\section{Case report}

A 10-year-old boy diagnosed at the Rheumatology Clinic with recurrent iritis over a period of 2 months was sent to the Department of Gastroenterology, Hepatology, Nutrition Disorders and Paediatrics in the Children's Memorial Health Institute with a suspicion of autoimmune hepatitis (AIH). During the medical check-up, the rheumatologist diagnosed hepatomegaly. Additional diagnostics revealed an elevated level of aminotransferases, gamma-globulins and positive ANA autoantibodies.

Laboratory tests, performed on admission (October 2008), revealed an elevated concentration of total and direct bilirubin $(2.27 / 1.55 \mathrm{mg} / \mathrm{dl})$, hypertransaminasaemia (AST $310 \mathrm{U} / \mathrm{l}$, ALT $348 \mathrm{U} / \mathrm{l}$ ), increased values of gamma-glutamyltransferase (GGTP $392 \mathrm{U} / \mathrm{l}$ ), gamma-globulin $(21 \mathrm{~g} / \mathrm{l})$ and $\operatorname{IgG}(21.2 \mathrm{~g} / \mathrm{l})$. Positive ANA antibodies 1: 5120 with a homogeneous type of lighting and anti-smooth muscle antibodies (ASMA) $1: 160$ were detected. An ultrasound scan of the abdomen showed an enlarged, heterogeneous liver with increased echogenicity. The first histopathological examination of a liver biopsy revealed severe inflammation in the portal tracts and necroinflammatory activity in the lobules. Inflammatory infiltrates consisted of lymphoid and plasma cells. Bridging fibrosis was observed in nearly all portal tracts. The scoring according to Batts and Ludwig's system was G3/S4 (Fig. 1A).

The diagnosis of AIH was confirmed and treatment with corticosteroid and azathioprine was introduced, which led to a decrease in liver enzymes (AST $50 \mathrm{U} / \mathrm{l}$, ALT $64 \mathrm{U} / \mathrm{l})$.

After 2 years of treatment, an increase in transaminase and gamma-glutamyltransferase levels was noted (AST 105 U/l, ALT 102 U/l, GGTP 193 U/l), while the concentration of bilirubin was normal. Therefore, a second liver biopsy was performed (October 2010), which revealed similar severe necroinflammatory activity, but the lymphoid cells also infiltrated the walls of the venous vessels and bile ducts. The presence of characteristic concentric periductal fibrotic rings suggested the component of overlap syndrome with PSC (Figs. 1B and C).

An increased dose of corticosteroid and azathioprine resulted in an improvement in laboratory results (AST 53 U/l, ALT 61 U/l). Treatment with ursodeoxycholic acid (UDCA) was added. Magnetic resonance cholangiopancreatography (MRCP) was performed in March 2011 and showed that the intrahepatic bile ducts were widened to $3 \mathrm{~mm}$, at places with stenosis. The choledochal duct and common hepatic duct were not widened.

The patient required another intensification of treatment in October 2011, because of an elevated activity of aminotransferases (AST 165 U/l, ALT 112 U/l) and an increase in total and direct bilirubin values
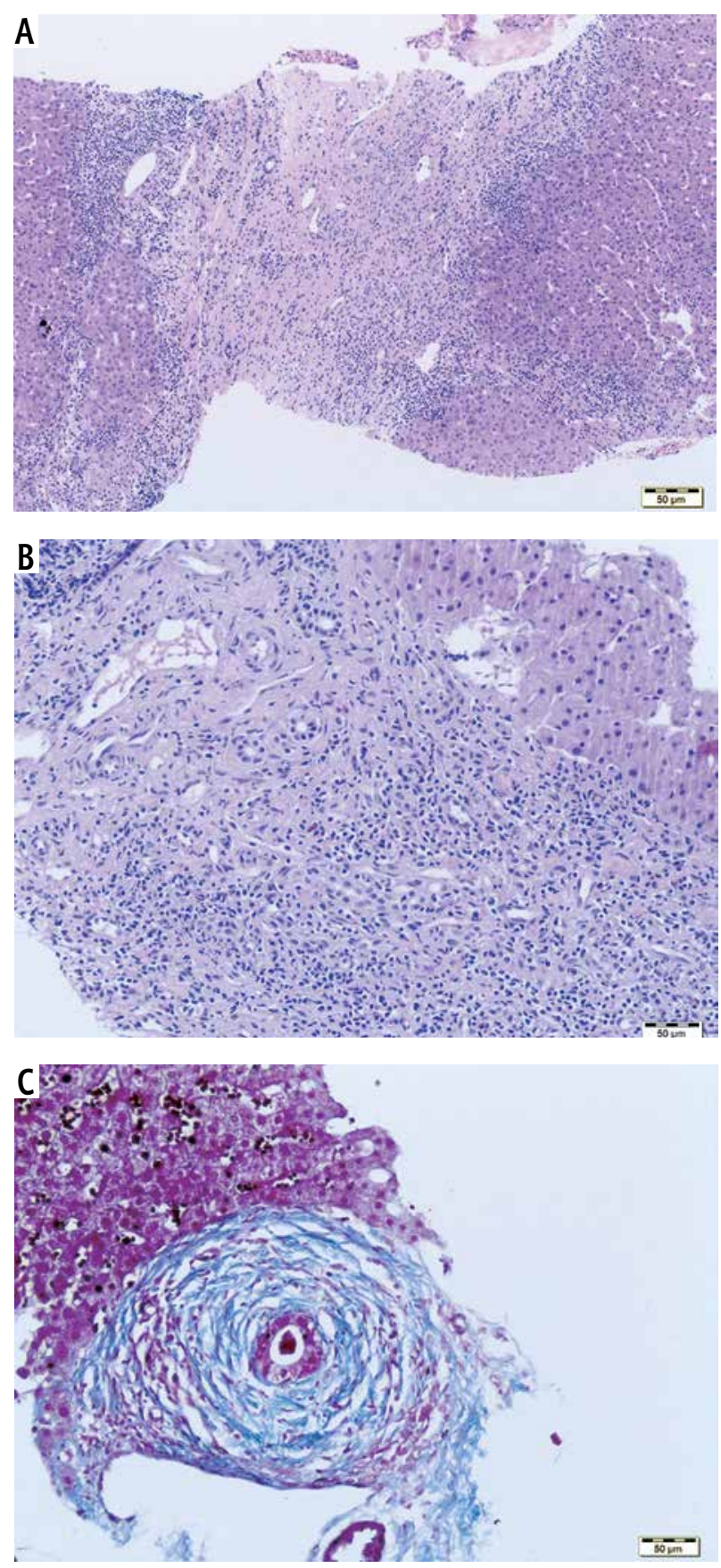

Fig. 1. A) Bridging fibrosis with severe necroinflammatory activity, H\&E. B) Severe lymphoid and plasma cell infiltration in the portal tract, mild periductal fibrosis, H\&E. C) Concentric periductal fibrosis Azan stain 
$(1.55 / 1.37 \mathrm{mg} / \mathrm{dl})$. An increased dose of corticosteroid and azathioprine again resulted in an improvement in laboratory tests.

In February 2012, the patient had his first episode of bleeding, from the upper gastrointestinal tract. Gastroscopy showed oesophageal varices grade II/III (according to the Paquet classification), and endoscopic varices ligation (EVL) was performed. Endoscopic varices ligation procedures were repeated three times within the next six months in order to achieve eradication of oesophageal varices.

Due to an increased risk of inflammatory bowel diseases (IBD) in the course of primary illness and a tendency to anaemia, colonoscopy was performed in July 2012. It showed a decreased vascular pattern in the sigmoid and rectum - an image corresponding to ulcerative colitis. Treatment with mesalazine was included.

At the age of 15 (in June 2013), after approximately a year of the 5-aminosalicylate treatment, the patient was admitted to the hospital emergency unite due to diarrhoea, severe abdominal pain, a general deterioration of his condition and jaundice. Laboratory tests revealed a significant increase in the concentration of total and direct bilirubin $(10.5 / 9.9 \mathrm{mg} / \mathrm{dl})$. The transaminases and gamma-glutamyltransferase concentrations were again elevated as before (ALT $80 \mathrm{U} / \mathrm{l}$, AST $80 \mathrm{U} / \mathrm{l}$, GGTP $270 \mathrm{U} / \mathrm{l}$ ). The albumin concentration was normal and the INR was slightly increased (1.27). The value of C-reactive protein (CRP) was increased $-5 \mathrm{mg} / \mathrm{dl}$ (normal range $<0.5 \mathrm{mg} / \mathrm{dl}$ ). A physical examination revealed hepatomegaly $(+4 \mathrm{~cm})$ and a very large splenomegaly (the spleen was entering the ileum). The imaging tests revealed fluid in the peritoneal cavity and the pleural cavities.

The patient was placed on the active list for a liver transplantation (LTX) due to a significant deterioration in liver functions.

Bacteriological tests of faeces showed no pathogenic flora. Sigmoidoscopy showed haemorrhoids and a decreased vascular pattern. Histopathological examination showed no abnormalities.

During this hospitalization, the patient reported very severe abdominal pain. Although wide spectrum diagnostics were performed, the cause was not identified. It was assumed that it may be a result of pressure on the internal organs by the enlarged spleen. After a surgical consultation, the patient was scheduled for a splenectomy, which was performed in July 2013. However, after surgery, the boy still reported abdominal pain, and periodically suffered from diarrhoea and vomiting with bile. Numerous analgesia modifications did not bring satisfactory results. It was observed that during a pain attack, there was no tachycardia or muscular guarding. Sometimes the pain disappeared after a placebo. These facts indicated a significant psychogenic component to these symptoms. After the diarrhoea ceased and the results of laboratory tests improved, the patient was discharged.

After two days of being at home, the boy was again admitted to the hospital, as an emergency. He complained of severe abdominal pain, had diarrhoea and vomiting. The laboratory tests showed a rapid deterioration of liver function (increased bilirubin concentration, coagulation disorders), deterioration of renal function and elevated inflammatory parameters: total bilirubin/direct $8.7 / 5.7 \mathrm{mg} / \mathrm{dl}$, INR 1.77, fibrinogen $1.7 \mathrm{~g} / \mathrm{l}$, ammonia $178 \mu \mathrm{g} / \mathrm{dl}$, albumin $21.9 \mathrm{~g} / \mathrm{l}$, GGTP $1,390 \mathrm{U} / \mathrm{l}$, creatinine $2.51 \mathrm{mg} / \mathrm{dl}$, urea $1.44 \mathrm{mg} / \mathrm{dl}$, CRP $27 \mathrm{mg} / \mathrm{dl}$.

From the beginning of exacerbation of the illness in June 2013, colonoscopy was performed twice and revealed a decreased vascular pattern and haemorrhoids. A histological examination showed no abnormalities. Gastroscopy showed oesophageal varices grade I and stomach fundus varices. An abdominal ultrasound scan revealed certain irregularities, a significantly thickened intestinal wall $(5-8 \mathrm{~mm})$, very poor peristalsis and conglomerates of compacted bile in the gallbladder. Magnetic resonance imaging of the abdomen revealed no signs of cancer. A neuroendocrine tumour (NET) was excluded as well: a scintigraphy with somatostatin analogues and a twenty-four hour collection of urine with an assessment of urinary catecholamines and their metabolites were normal. A computed tomography (CT) scan of the abdomen vascular option (angiographic CT) revealed no abnormalities of blood supply to the intestinal wall. At the same time, the repeated bacteriological tests showed no increase in pathogenic flora.

During 2 months of hospitalization, two episodes of massive gastrointestinal bleeding occurred. The first was bleeding from the lower gastrointestinal tract which caused a decrease of concentration of haemoglobin down to $3.6 \mathrm{~g} / \mathrm{dl}$. Colonoscopy revealed the presence of thrombus at the splenic flexure (the probable source of bleeding) and the presence of a long clot in the sigmoid colon. The mucous membrane of the colon had a decreased vascular pattern, with a single erosion. Gastroscopy revealed no signs of active or previous bleeding.

After a week, there was a second episode of massive bleeding in the upper and lower gastrointestinal tract. The patient was transferred to the Intensive Care Unit. After initial stabilization of his general condition, a liver transplantation from a cadaveric donor was performed with Y-Roux anastomosis. The transplant was 
performed according to standard techniques. Basiliximab, tacrolimus, mycophenolate mofetil and corticosteroids were introduced for immunosuppression induction. The post-surgery period was complicated by rectal bleeding. Colonoscopy revealed inflammation with several superficial ulcers and a lack of vascular pattern. Additionally, a positive Clostridium difficile culture was obtained. Treatment with vancomycin and later with fidaxomicin was started and tacrolimus was switched with cyclosporine. Five weeks after the transplantation the patient was discharged with good liver function. During three years of observation his liver function has been excellent; he is on triple immunosuppressive therapy (with a small dose of corticosteroids), oral mesalamine and UDCA.

\section{Discussion}

Cirrhotic patients may have stable liver function for a long period. Unfortunately, any acute insult in a patient with decreased liver function may lead to a sudden hepatic decompensation [7]. In contrast to the natural history of progression of cirrhotic patients leading to irreversible "end stage" liver diseases, in ACLF there is an element of reversibility and some patients may recover when they receive aggressive intensive care support.

Unfortunately, there are no ways to foresee the future for a patient suffering from ACLF. Jalan et al. in a group of 497 cirrhotic patients, of whom 180 developed ACLF, observed a 53\% mortality rate [6]. Patients with infections had the worst overall prognosis $(p<0.05)$. They also showed that patients who had been hospitalized within the last 6 month had a higher mortality rate than those without a recent hospitalization $(78 \%$ vs. $34 \%)$. It is well known that cirrhotic patients, due to depression of the reticuloendothelial system, dysfunction of the cellular immune system and defects of the bacterial translocation, are more susceptible to severe infections and sepsis [8]. Although in our patient we did not find the exact precipitating infection factor, the clinical course of the illness (diarrhoea, severe abdominal pain) with quick deterioration suggests this.

The available literature is sparse, with regard to ACLF in children. Most papers report the outcome of hepatitis E virus (HEV) or hepatitis A virus (HAV) superinfection over CLD in developing countries [9]. The most common clinical feature was jaundice $(94.4 \%)$ and ascites (69\%).

ACLF patients may have varied manifestation due to heterogeneity in the patient population (different underlying chronic liver disease, different acute insult). The causes of chronic liver damage vary accord- ing to geographic location. In the adult population, they mostly include chronic viral hepatitis, alcohol abuse, autoimmune diseases, and nonalcoholic fatty liver disease, while in the paediatric population, they are mostly cholestatic and metabolic liver diseases [10]. Precipitating events, worsening liver function, can be divided into two groups [7]. The first involves events directly affecting liver function such as drug-induced liver injury, new-onset viral hepatitis, alcoholic and ischaemic hepatitis. The second involves extrahepatic, systemic events, which have an influence on liver functions such as infection, surgery, trauma or variceal bleeding.

Autoimmune liver diseases are a more common indication for a liver transplantation in adult patients than in children [10]. Unfortunately, in children and young adults $\mathrm{AIH}$ presents a more aggressive course and more often has overlap syndrome with primary sclerosing hepatitis with not only interface hepatitis, but also bile duct damage [11]. Patients with a histological diagnosis of PSC, but without the typical intraand/or extrahepatic cholangiographic changes, have been identified as having small duct PSC. The most common criteria for a diagnosis of small duct PSC that have been proposed are the occurrence of biochemical features of chronic cholestasis, liver histology compatible with PSC, normal cholangiogram, and concomitant IBD. The prevalence of IBD in patients with PSC has been reported as ranging from $71 \%$ to $81 \%$ [12]. The most common symptoms in small duct patients were fatigue and abdominal pain.

In our patient, the first biopsy was consistent with AIH diagnosis, but the second also showed the features of PSC. Although in some children (>44\%) cirrhosis is present at diagnosis, mortality is low and most children remain clinically stable for a long period. Development of end stage liver disease, requiring liver transplantation, is reported in less than $10 \%$ of children, mostly in patients who were diagnosed 10-14 years earlier [13]. Children with AIH/PSC overlap syndrome more commonly had concurrent inflammatory bowel disease, as was the case with our patient.

In pathophysiology of ACLF, a systemic inflammatory response and neutrophil dysfunction play a crucial role $[2,3]$. Wasmuth et al. reported the similarity of ACLF and septic shock, characterised by progressive vasodilatation and multiple organ failure [8].

Still, is not defined whether the prognosis of the patient depends on the degree of acute insult, chronic insult, or a combinations of both. According to the Jalan study [6] the type of insult may have important prognostic value, and infection was associated with the worst prognosis $(p<0.05)$. 
Some patients with ACLF may benefit from the molecular adsorbent recirculating system (MARS), which gives time for recovery or serves as a "bridge" to transplantation [5]. However, in a meta-analysis study MARS did not significantly reduce mortality compared with standard medical treatment [14]. In the long-term perspective liver transplantation is the only curative option that can save a patient with ACLF. Unfortunately, there is a high (54\%) mortality rate amongst patients waiting for a transplantation [15].

\section{Conclusions}

1. Acute-on-chronic liver failure occurs in patients with chronic liver disease who suffer from an acute insult and deterioration of previously compensated liver disease.

2. The mortality rate among patients with ACLF is higher than in patients with CLD awaiting liver transplantation.

3. Multisystem organ failure is the primary cause of death in ACLF.

4. Less than $50 \%$ of ACLF patients have a chance of recovery during the critical period if they receive aggressive intensive care support, but liver transplantation is the only curative option.

\section{Disclosure}

The authors report no conflict of interest.

\section{References}

1. Ohnishi H, Sugihara J, Moriwaki H, et al. Acute-on-chronic liver failure. Ryoikibetsu Shokogun Shirizu 1995; 7: 217-219.

2. Olson JC, Kamath PS. Acute-on-chronic liver failure: concept, natural history and prognosis. Curr Opin Crit Care 2011; 17: 165-169.

3. Sarin SK, Kumar A, Almeida JA, et al. Acute-on-chronic liver failure: consensus recommendation of the Asian Pacific Association for the study of the liver (APASL). Hepatol Int 2009; 3 : 269-282.

4. Wicher D, Nalepa A, Jankowska I, et al. Ostre uszkodzenie w przebiegu przewlekłej choroby wątroby. Standardy Med Pediatria 2014; 11: 533-539.

5. Sen S, Davies NA, Mookerjee RP, et al. Pathophysiological effects of albumin dialysis in acute-on-chronic liver failure: a randomized controlled study. Liver Transpl 2004; 10: 1109-1119.

6. Jalan R, Stadlbauer V, Sen S. Natural history of acute decompensation of cirrhosis: the basis of the definition, prognosis, and pathophysiology od acute-on-chronic liver failure. Hepatology 2006; 44 (Suppl 1): 371A-372A.

7. Nava LEZ, Valadez JA, Chavez-Tapia NC, et al. Acute-on-chronic liver failure: a review. Therapeutics Clin Risk Manegement 2014; 10: $295-303$.
8. Wasmuth HE, Kunz D, Yagmur E, et al. Patients with acute on chronic liver failure display "sepsis-like" immune paralysis. J Hepatol 2005; 42: 195-201.

9. Jagadisan B, Srivastava A, Yachha SK, et al. Acute on chronic liver disease in children from the developing world: recognition and prognosis. JPGN 2012; 54: 77-82.

10. Pawlak J, Pawłowska J. Transplantacje wątroby: analiza biorców w ośrodku pediatrycznym i dla dorosłych. Med Sci Rev 2002; 2: $135-138$.

11. Mieli-Vergani G, Vergani D. Autoimmune hepatitis in children: What is different from adult AIH. Semin Liver Dis 2009; 29: 297-306.

12. Björnsson E, Boberg KM, Cullen S, et al. Patients with small duct primary sclerosing cholangitis have a favourable long term prognosis. Gut 2002; 51: 731-735.

13. Gregorio GV, Portman B, Reid F, et al. Autoimmune hepatitis in childhood: a 20-year experience. Hepatology 1997; 25: 541-547.

14. Khuroo MS, Khuroo MS, Farahat KL. Molecular adsorbent recirculating system for acute-on-chronic liver failure: a meta-analysis. Liver Transplant 2004; 10: 1099-1106.

15. Finkenstedt A, Nachbaur K, Zoller H, et al. Acute on chronic liver failure: excellent outcomes after liver transplantation but high mortality on the wait list. Liver Transplant 2013; 19: 879-886. 